\title{
RTBIMS: Accuracy Enhancement in Iterative Multiplication Strategy for Computing Propagated Trust
}

\author{
Hassan Shakeri \\ Department of Computer \\ Ferdowsi University of Mashhad \\ Mashhad - Iran \\ shakeri@mshdiau.ac.ir
}

\author{
Abbas Ghaemi Bafghi \\ Department of Computer \\ Ferdowsi University of Mashhad \\ Mashhad - Iran \\ ghaemib@um.ac.ir
}

\begin{abstract}
Trust Management (TM) systems are frameworks for managing security in decentralized environments. However computing the trust value is a challenge when the source entity has no experience of previous direct interaction with the sink. A popular way to estimate the trust value in this case is trust propagation. It suggests evaluating the trust value between two nodes based on the information received from intermediate nodes between them. As we often use a number between 0 and 1 to represent the trust value, the Iterative Multiplication Strategy (IMS) is usually used to estimate the value of propagated trust. In this paper we introduce RTBIMS, an accuracy-enhanced version of IMS. In our algorithm, we first calculate recommendation trust for each intermediate node based on its trustworthiness in judgments and similarity between its views and the source ones. We then use these values in the multiplication chain. We have examined RTBIMS on a popular trust dataset (Advogato) and the results show that its accuracy in evaluating trust is much higher than the basic IMS.
\end{abstract}

Keywords- trust, trust propagation, web of trust, trust chain, iterative multiplication strategy (IMS), RTBIMS

\section{INTRODUCTION}

There are many cases in computer systems where a node (a person or a system) needs to evaluate the trustworthiness of another node. For instance, in computer network routing, to decide on the best route, a node should be aware of the trustworthiness of the nodes in the path. In agent-based systems, both the platform and the agent should have an estimation of the other party's trustworthiness. On Internet, users and web sites need to evaluate trustworthiness of the other users and web sites. An access control system should be decide in what capacity a user who requests for a particular service is trustworthy. Furthermore, trust has a key role in many other fields such as semantic web, game theory, decision making in the uncertainty circumstances, etc. However there are remarkable differences in the concept, representation, computing and using trust in these different fields [3].

Among different definitions of trust, three of them are the most popular:
The first definition from Mui et al. [16] refers to past encounters, and may be thought of by some as "reputationbased" trust:

"[Trust is] a subjective expectation an agent has about another's future behavior based on the history of their encounters."

The next definition, from Grandison and Sloman [8] introduces context and is unique in referring to the "competence" to act (instead of actions, themselves):

"[Trust is] the firm belief in the competence of an entity to act dependably, securely, and reliably within a specified context."

The third definition, from Olmedilla et al. [17] applies to many cases and unlike the previous definition, refers to actions and not competence:

"Trust of a party A to a party B for a service $X$ is the measurable belief of $A$ in that $B$ behaves dependably for $a$ specified period within a specified context (in relation to service $\mathrm{X})$."

As a general classification, there are two approaches to evaluate the value of trust: policies and reputation. Policies describe the conditions necessary to obtain trust, and can also prescribe actions and outcomes if certain conditions are met. Policies frequently involve the exchange or verification of credentials, which are information issued (and sometimes endorsed using a digital signature) by one entity, and may describe qualities or features of another entity. For example, having the credential of a university degree means its holder has been recognized by the issuing university as having a certain education level [3].

Reputation is an assessment based on the history of interactions with or observations of an entity, either directly with the evaluator (personal experience) or as reported by others (recommendations or third party verification). How these histories are combined can vary, and recursive problems of trust can occur when using information from others. At a basic level, both credentials and reputation involve the transfer of trust from one entity to another, but each approach has its 
own unique problems which have motivated much of the existing work in trust [3].

There are two ways to evaluate trust value based on reputation in turn: centralized and decentralized. In the centralized approach there exists a trusted third party who can evaluate each node's reputation based on its history. However the researchers often prefer the decentralized approach for reputation management $[1,2]$.

A trust decision can be a transitive process, where trusting one piece of information or information source requires trusting another associated source. For example, one might trust a book and its author because of the publisher, and the publisher may be trusted only because of the recommendation of a friend.

Reputation is considered as a trust measure. Each entity maintains reputation information on other entities, thus creating a "web" that is called web of trust.

If there is no link between a pair of entities, it means no trust decision has yet been made. This is the case in which trust transitivity can be applied, a simplified example being if A trusts $\mathrm{B}$ and $\mathrm{B}$ trusts $\mathrm{C}$, then $\mathrm{A}$ trusts $\mathrm{C}$. This property is also known as trust propagation. However there is discussion on how much transitivity is valid and which formula or algorithm should be used for evaluating propagated trust value in each field. Multiple researchers are exploring ways to transfer trust within a web of trust. We refer for example to $[12,18,21]$.

Trust if often represented as a real value between 0 and 1 . To estimate the value of the propagated trust, one simple and popular idea is to use iterative multiplication strategy (IMS). We describe this strategy using an example: Let $\mathrm{A}$ has no experience of previous interaction with $\mathrm{C}$ (and so no direct knowledge on C's trustworthiness value). However let the amounts of trust that $\mathrm{A}$ holds for $\mathrm{B}$ and $\mathrm{B}$ holds for $\mathrm{C}$ are 0.8 and 0.5 respectively. We can infer the value of trust that $A$ can hold for $\mathrm{C}$ using simple multiplication as $0.8 * 0.5=0.4$. We may generalize this idea so that if there is a path between $X$ and $\mathrm{Y}$ in the web of trust, we can use this path as a chain of trust and estimate the value of trust that $X$ should hold for $Y$ by multiplying the trust labels of the links on this chain.

In this paper we illustrate that it is important to distinct between competence trust and recommendation trust in using iterative multiplication strategy. Based on this idea, we propose RTBIMS (Recommendation-Trust Based Iterative Multiplication Strategy), an accuracy-enhanced version of IMS. In our algorithm, we first calculate recommendation trust for each intermediate node based on its trustworthiness in judgments and similarity between its views and the source ones. We then use these values in the multiplication chain.

The rest of this paper is organized as follows: Section 2 describes related work. In section 3 we introduce our new algorithm, RTBIMS. Section 4 reports the results of experiments. Finally we conclude the paper and present possible future research in section 5 .

\section{RELATED WORK}

Many researchers have worked on the algorithms for how trust is transferred, combined, or resolved. In [20, 21] a set of hypotheses and experiments are described for testing how trust is transferred between hyperlinks on the Web. Specifically, this work examines how much trust (in the context of a consumer trusting a business for purchasing a product) is transferred from a trusted web resource to an unevaluated one. The transfer is evaluated considering differing types of links, types of resources, and types of trust in the known source.

Other more recent works look at how to compute trust transitivity given actual quantities for trust or distrust. A key work in this area is [18] whose goal is to provide a means of merging trust that is robust to noise. Emphasizing personalized trust, as opposed to globally computed values, this approach is described as a generalization of PageRank [6] to the Semantic Web.

In [13] the problem of controversial users (those who are both trusted and distrusted) is addressed. This work shows that the globally computed trust value (in a web of trust) for a controversial user may not be as accurate as a locally computed value due to the global disagreement on trust for that user.

A difficult problem addressed in [19] is the transitivity of distrust, the main problem being if $\mathrm{A}$ distrusts $\mathrm{B}$ and $\mathrm{B}$ distrusts $\mathrm{C}$, we cannot say if A trusts $\mathrm{C}$. This work also evaluates and ranks several methods for propagating trust and distrust in a given web of trust.

In several models of access control for pervasive environments (including [11] and [19]), propagated trust is computed by iteratively multiplying the trust values on the path from a source entity to the target entity (Iterative Multiplication Strategy).

Ding et al. in [7] classified trust into two broad categories:

- Referral Trust: Trust to the other's knowledge in a particular domain

- Associative Trust: the value of similarity between two agents' ideas.

Then they identified five types of trust, one of which, STT is used in our algorithm. STT stands for Similar Trusting Trust and is an associative trust that evaluates the similarity of two agents' trust knowledge. STT $_{\mathrm{ijd}}$ refers to the similarity of agent $a_{i}$ and agent $a_{j}$ 's referral trust to the other agents within domain d. Intuitively STT clusters trustors (agents who maintain trust knowledge). This paper formalizes trust network inference notions, providing both data and computational models, and suggests an evaluation model for benchmarking.

Some works including [14, 15] addressed the concept of disposition to trust and its importance in trust management. Disposition to trust is the inherent propensity of an individual to trust or distrust others. An individual's disposition to trust is a stable characteristic of his/her personality that governs how he/she views the trustworthiness of every other entity that he/she encounters. A study in the context of ecommerce by McCord and Ratnasingam [14] has demonstrated that there is a strong relationship between an individual's disposition to trust 
and the trust related decisions that they make. Based on this, Hasan et al. in [10] addressed a challenge in trust propagation: how to use trust propagation without the negative effect of subjectivity. They used a method for eliminating subjectivity from trust recommendations.

Hasan et al. also in [11] evaluated the effectiveness of iterative multiplication for trust propagation using the dataset of Advogato which is a real web of trust.

\section{OUR Proposed AlgORITHM: RTBIMS}

Trust propagation is based on the transitivity property of trust. In a general case we may express this property by:

$$
\mathrm{T}_{\mathrm{A}, \mathrm{C}}=\mathrm{T}_{\mathrm{A}, \mathrm{B}} \text { o } \mathrm{T}_{\mathrm{B}, \mathrm{C}}
$$

Where $O$ is a concatenation operator, which is multiplication when we use iterative multiplication strategy (IMS). In other words, according to iterative multiplication strategy, in a web trust, if node $\mathrm{v}_{1}$ has no experience of previous interaction with node $\mathrm{v}_{\mathrm{n}}$ (and thus no direct information on its trustworthiness), but there is a path $v_{1} v_{2} \ldots v_{n}$ so that all the labels of the links on the path are known, $v_{1}$ can consider this path as a trust chain and estimate $\mathrm{v}_{\mathrm{n}}$ 's trustworthiness by multiplying the labels of all links on the chain [10]. Therefore if we denote the value of trust that $v_{i}$ holds for $v_{j}$ by $T_{i, j}$, we will have

$$
T_{1, n}=T_{1,2} * T_{2,3} * \ldots * T_{n-1, n}=\prod_{i=1}^{n-1} T_{i, i+1}
$$

This is the generalization of the formula

$$
\mathrm{T}_{\mathrm{A}, \mathrm{C}}=\mathrm{T}_{\mathrm{A}, \mathrm{B}} * \mathrm{~T}_{\mathrm{B}, \mathrm{C}}
$$

As an example, Fig. 1 depicts a chain of trust with the values $0.7,0.5$ and 0.8 . Using (3), the source (x) might infer its trust of 0.28 to the sink (w).

In this section we introduce our new algorithm, RTBIMS. First, we suggest a new formula for propagated trust. Then we describe our way to estimate recommendation trust from competence trust values which is needed in the cases where recommendation trust is not available directly. Finally we address the cases where multiple paths exist from source to sink and we use an example to demonstrate the whole algorithm.

\section{A. New Way to Estimate Propagated Trust}

We know that in (3) $T_{A, B}$ denotes the amount of trust that $A$ holds for $\mathrm{B}$. In many applications, this value represents the value of A's belief on $B$ 's competence in a specific domain.

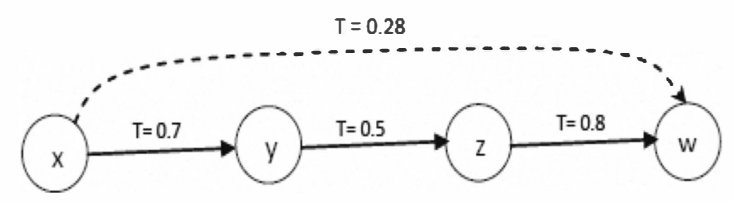

Figure 1. Trust propagation using IMS: Propagated trust is simply estimated by multiplying the trust values of edges in the path.
However in (3) this value is used as a measure of the correctness of $B$ 's judgment and recommendation about $\mathrm{C}$. These two concepts are not necessarily the same. In other words, we should distinct between A's belief on $B$ 's competence and on validity of $B$ 's recommendations about a third party.

Although in many applications, a person who has more competence in a particular domain also provides more accurate recommendations about another person's competence in that domain, this is not true in all areas and applications equally. In many applications, people who have themselves little competence to perform a task still can offer good recommendations about the competence of other people for that task. On the other hand, there are cases where a person has high competence to perform a task, but his judgment and recommendation about other people's competence is not so reliable.

So we suggest two types of trust to be considered:

- Competence Trust: the value of A's belief in that B has competence to perform a task in a specific domain.

- Recommendation Trust: the value of A's belief in that B's recommendations about the competence of third parties are correct and reliable.

We will denote these two types of trust by CT and RT, respectively. According to the above discussion, to compute indirect trust from $\mathrm{A}$ to $\mathrm{C}$, we suggest to modify the formula (3) to obtain (4).

$$
\mathrm{CT}_{\mathrm{A}, \mathrm{C}}=\mathrm{RT}_{\mathrm{A}, \mathrm{B}} * \mathrm{CT}_{\mathrm{B}, \mathrm{C}}
$$

Often the values of CT are available in our web of trust. However the values of RT should be estimated. Comparing the above definition of RT and the definition of STT in [7] which we mentioned in the previous section, we suggest using STT as an estimation of RT. Based on the transitivity of STT we can conclude (5).

$$
\mathrm{RT}_{\mathrm{A}, \mathrm{C}}=\mathrm{RT}_{\mathrm{A}, \mathrm{B}} * \mathrm{RT}_{\mathrm{B}, \mathrm{C}}
$$

Now we can combine (4) and (5) to obtain a general formula to be used in a web of trust:

$$
\begin{aligned}
C T_{1, n}=R T_{1,2} * R T_{2,3} * \ldots * C T_{n-1, n} \\
=\prod_{i=1}^{n-2} R T_{i, i+1} * C T_{n-1, n}
\end{aligned}
$$

As (6) shows, in the multiplication of trust values of the chain links, only the last factor is of type competence trust. It represents the value of trust on the last node's competence according to his previous node's judgment. The other factors are of type recommendation trust, because they should express the degree of correctness of each node's judgment about its next node.

\section{B. Estimating Recommendation Trust}

If the values of competence trust and recommendation trust are available independently, we can use (6) directly. However in many cases we have only the values of competence trust. As 
we mentioned before, in such cases we may compute STT as the estimation of RT: we use the degree of similarity between A's and B's recommendations about other entities as a measure of recommendation trust that $\mathrm{A}$ can hold for $\mathrm{B}$. So we need to compute the similarity matrix. A similarity matrix is a matrix of scores which express the similarity between two data points. Alternatively we can compute the distance matrix which is a matrix containing the distances, taken pairwise of a set of points. Since the most natural metric for computing distance in most applications is the Euclidean metric [5], we use the Euclidean distance matrix. In this matrix, the distance between two points $i$ and $j$ in n-dimensional space is computed as (7).

$$
D_{i, j}=\sqrt{\sum_{k=0}^{n}\left(x_{i k}-x_{j k}\right)^{2}}
$$

\section{Decision on Multiple Paths}

When there is more than one path from $v_{1}$ to $v_{n}$, we may compute the final trust based on either maximum or average of the results from different paths or we can use another way of combination. Choosing one of these approaches depends on the application as well as the source disposition to trust. For example if the source entity has a high disposition to trust, it is more suitable to use the maximum value among different paths rather than the average of them.

Fig. 2 depicts an example of using (6) considering

a) the maximum of different path values as the final estimated trust value $\left(\mathrm{T}_{\max }\right)$, and

b) the average of different path values as the final estimated trust value $\left(\mathrm{T}_{\mathrm{avg}}\right)$.

\section{EXPERIMENTS}

To evaluate the accuracy of estimations made by RTBIMS and comparing it with the basic iterative multiplication algorithm (IMS), we examined both algorithms on the dataset of Advogato. Advogato contains trust information between members of an internet forum of programmers and one of its main aims is to provide data for experiments in the trust domain. Some research works including [11] used this dataset for such experiments.

\section{A. Dataset Characteristics}

Advogato dataset is a large text file that currently contains 71,000 rows of text data representing trust information between about 14,000 programmers.

The file begins with the phrase "digraph $G$ \{ " at the first line and ends with the " $\}$ " at the last line. In the middle lines trust information of each programmer to the other programmers begins with that programmer's name enclosed in " $/ * * /$ ". In the following lines, the amount of the programmer's trust to other programmers is specified with one of the words "Master", "Journeyer", or "Apprentice".

A part of the dataset is shown in Fig. 3.

For example, in the part of dataset shown in Fig. 3, the first programmer who has judged about others is dbarth. He has evaluated mikl as a journeyer programmer. The next one is minami who has evaluated himself as apprentice but polo, movement, and jao as journeyer programmers. In the same way, polo and other programmers have expressed their judgments about others.

The words "Master", "Journeyer", and "Apprentice" should be interpreted as numbers between 0 and 1 in a suitable way. The site of Advogato itself suggests the numbers 1, 0.8, and 0.6 as the equivalents for the three words respectively.

\section{B. Experiment Results}

We used a computer program to evaluate the estimation accuracy of the proposed algorithm of RTBIMS, and to compare RTBIMS with the iterative multiplication algorithm (IMS). The program first reads the data from dataset and forms a web of trust based on it. From implementation point of view, the web of trust, which is a directed graph, is represented as a matrix. The elements of this matrix denote the value of competence trust between programmers.

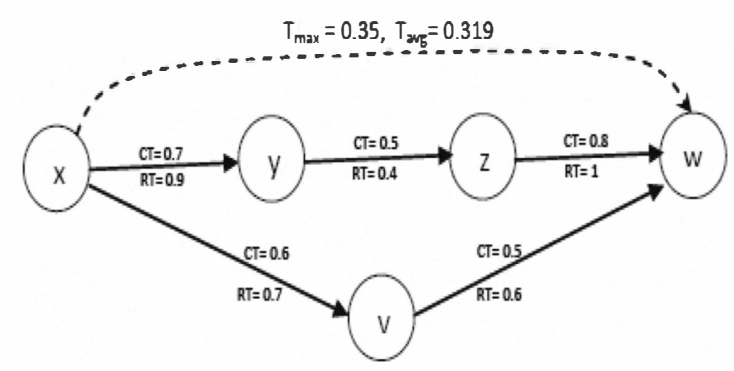

Figure 2. Trust propagation using RTBIMS: $\mathrm{T}_{\max }$ is the maximum and $\mathrm{T}_{\mathrm{avg}}$ is the average of the estimated trust using diferent paths.

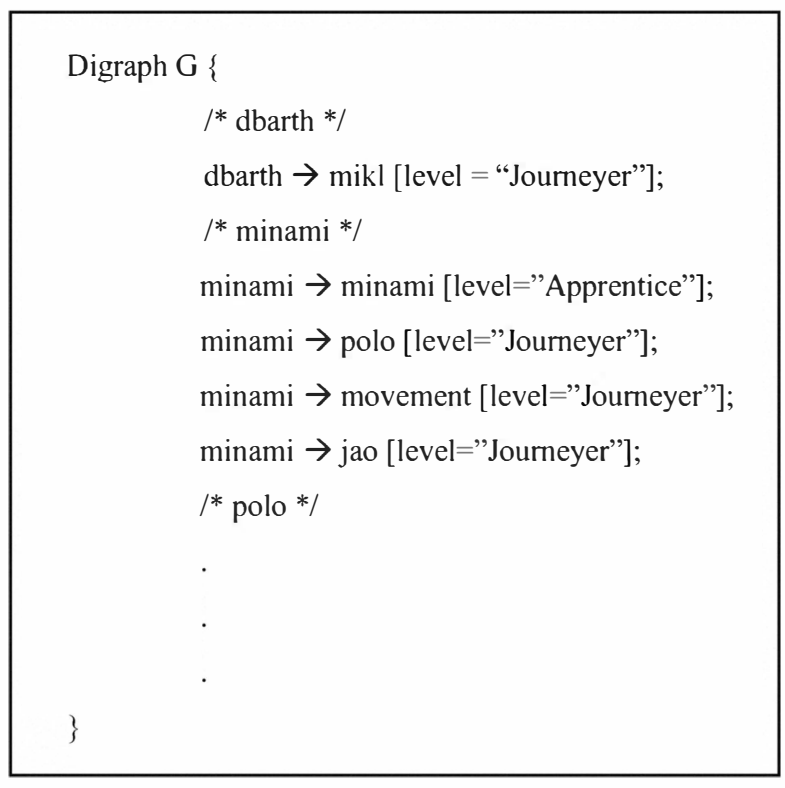

Figure 3. A part of Advogato dataset: For example "dbarth" has evaluated "mikl" as a journeyer programmer. 
To estimate the values of recommendation trust between programmers, we calculated the degree of similarity between their opinions (recommendations) about other programmers: for any two programmers $p_{i}$ and $p_{j}$, we extracted their recommendation list about other programmers and computed the similarity between the two lists using (7). We used the resulting value as the recommendation trust between two programmers.

Three types of experiments were directed:

1- Using IMS algorithm

2- Using RTBIMS considering the maximum value among results from different paths

3- Using RTBIMS considering the average of results from different paths

To evaluate the results, we used the Leave-One-Out technique: we chose pairs that direct trust between them was available in the dataset and compared the list of these direct trust values with the propagated trust estimated for that pairs by each algorithm. For comparison, we computed three measures: correlation coefficient, average of differences, and average of absolute differences.

Table 1 shows the results of experiments. As this table shows RTBIMS provides a much higher correlation between direct (actual) and estimated trust values than IMS. However the difference between the correlation values is negligible when we use two different approaches in RTBIMS: using maximum or average among the results from multiple paths.

The next column depicts the average of differences between direct and estimated trust values (direct value minus estimated value). This value is very high in IMS, while is too lower in RTBIMS using maximum and is almost zero in RTBIMS using average.

Finally the last column depicts the average of absolute differences between direct and estimated trust values. It shows that RTBIMS provides a lower average of absolute differences than IMS. However like the correlation column, this value is almost the same for two approaches in RTBIMS.

On the other hand, comparing the two last columns of table 1 might be interesting. Remember that in the "Average of differences" column, we show the value of direct value minus estimated value. So in the first row of the two last columns, where we see almost equal values 0.24 and 0.25 , we may infer that IMS almost always estimates smaller values for trust comparing to actual values.

Comparing the values -0.06 and 0.06 in the next row, we infer that "RTBIMS using maximum" almost always estimates larger values for trust than actual values. Finally the value 0.003 (almost 0) in the last row shows that "RTBIMS using average" results the closest estimation to the actual values according to the average of differences.

Since IMS and RTBIMS use competence and recommendation trust respectively to compute propagated trust, we may infer from the above results and analysis that
1) Recommendation trust provides more accurate results in trust propagation than competence trust. This implies that people often recommend well even if they are not themselves competent enough in a domain.

2) Using competence trust results in lower estimated trust values comparing to the real values. This implies that if we use competence trust for estimating propagated trust, we will obtain pessimistic results.

\section{CONCLUSION AND FUTURE WORK}

It is popular to use iterative multiplication strategy to compute the trust value in trust propagation. However this strategy is not so accurate because it does not distinct between competence trust and recommendation trust. To address this problem, we have proposed a new algorithm, RTBIMS, that uses recommendation trust values in estimating propagated trust. We have also suggested a way to estimate the recommendation trust itself based on similarities.

In the future, we will work to further improve the accuracy of the estimation of propagated trust. We will also try to find ways for reducing the communication among nodes so that the algorithm will be practical for distributed systems. In addition, we will work on the case of multiple paths to determine the most accurate method to combine the results from different paths in different applications.

\section{TABLE I. RESULTS OF IMS AND RTBIMS ON ADVOGATO DATASET}

\begin{tabular}{|c|c|c|c|}
\hline Algorithm & Correlation & Average of differences & $\begin{array}{c}\text { Average of absolute } \\
\text { differences }\end{array}$ \\
\hline IMS & 0.61 & 0.24 & 0.25 \\
\hline RTBIMS using maximum & 0.73 & -0.06 & 0.06 \\
\hline RTBIMS using average & 0.75 & -0.003 & 0.05 \\
\hline
\end{tabular}

\section{REFERENCES}

[1] Abdul-Rahman, A. and Hailes, S. , A distributed trust model, In Proceedings of the New Security Paradigms Workshop, pages 48-60. ACM, 1997.

[2] Abdul-Rahman, A. and Hailes, S., Using recommendations for managing trust in distributed systems., In Proceedings of IEEE International Conference on Communication, 1997.

[3] Artz, D. and Gil, Y., A Survey of Trust in Computer Science and the Semantic Web, Journal of Web Semantics: Science, Services and Agents on the World Wide Web, 2007

[4] Bonatti, P., Duma, C., Olmedilla, D., and Shahmehri, N., An integration of reputation-based and policy-based trust management, In Proceedings of the Semantic Web Policy Workshop, 2005.

[5] Breu H., Gil J., Kirkpatrick, D., and Werman, M., Linear Time Euclidean Distance Transform Algorithms, IEEE transactions on Pattern Analysis and Machine Intelligence. vol. 17, no. 5, May 1995.

[6] Brin, S. and Page, L., . The anatomy of a large-scale hypertextual Web search engine, Computer Networks and ISDN Systems, 30(1-7):107$117,1998$.

[7] Ding, L., Kolari, P., Ganjugunte, S., Finin, T., and Joshi, A., Modeling and evaluating trust network inference. In Proceedings of the 7th International Workshop on Trust in Agent Societies at AAMAS, 2004.

[8] Grandison, T. and Sloman, M., A survey of trust in internet applications, IEEE Communications Surveys and Tutorials, 4(4):2-16, 2000 
[9] Guha, R., Kumar, R., Raghavan, P., and Tomkins, A., Propagation of trust and distrust. In WWW '04: Proceedings of the 13th international conference on World Wide Web, pages 403-412, New York, NY, USA, ACM Press, 2004.

[10] Hasan, O., Pierson, J. M. and Brunie, L., Access Control in Ubiquitous Environments Based on Subjectivity Eliminated Trust Propagation, In Proc of the $3^{\text {rd }}$ Intl. Conf. on Embedded and Ubiquitous Computing (EUC 2008), Shanghai, China, 2008

[11] Hasan, O., Brunie, L. and Pierson, J. M., Evaluation of the Iterative Multiplication Strategy for Trsust Propagation in Pervasive Environments, ICPS'09, London, United Kingdom, 2009.

[12] Massa, P. and Hayes, C., Page-rank: Using trusted links to re-rank authority, In WI'05: Proceedings of the 2005 IEEE/WIC/ACM International Conference on Web Intelligence (WI'05), pages 614-617, Washington DC, USA, IEEE Computer Society, 2005.

[13] Massa, P. and Avesani, P., Controversial users demand local trust metrics: an experimental study on epinions.com community, In Proceedsings of the 25th American Association for Artificial Intelligence Conference, 2005.

[14] McCord, M., and Ratnasingam, P., The impact of trust on the technology acceptance model in business to consumer e-commerce. In Proc. Int1. Conf. of the Information Resources Management Association: Innovations Through Information Technology, May 2004.

[15] McKnight, D. H., Choudhury, V., and Kacmar, C., Developing and validating trust measures for e-commerce: An integrative typology. Information Systems Research, 13(3):334 - 359, September 2002.

[16] Mui, L., Mohtashemi, M., and Halberstadt, A., A computational model of trust and reputation, In Proceedings of the 35th International Conference on System Science, pages 280-287, 2002.

[17] Olmedilla, D., Rana, O., Matthews, B., and Nejdl, W., Security and trust issues in semantic grids, In Proceedings of the Dagsthul Seminar, Semantic Grid: The Convergence of Technologies, volume 05271, 2005.

[18] Richardson, M., Argawal, R., and Domingos, P., Trust management for the semantic web, In Proceedings of the Second International Semantic Web Conference, pages 351-368, Spring-Verlag, 2003.

[19] Saadi, R., Pierson J.-M., and Brunie., L., Authentication and access control using trust collaboration in pervasive grid environment, In Proceedings of the International Conference on Grid and Pervasive Computing (GPC 2007), 2007.

[20] Stewart, K. J., Transference as a means of building trust in world wide web sites, In ICIS '99: Proceeding of the 20th international conference on Information Systems, pages 459-464, Atlanta, GA, USA. Association for Information Systems, 1999.

[21] Stewart, K. J. and Zhang, Y., Effects of hypertext links on trust transfer. In ICEC '03: Proceedings of the 5th international conference on Electronic commerce, pages 235-239, New York, NY, USA. ACM Press, 2003 